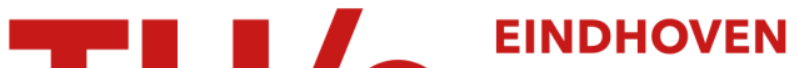 UNIVERSITY OF TECHNOLOGY
}

\section{Optical measurement of magnetic anisotropy in thin garnet films}

Citation for published version (APA):

Rijnierse, P. J., Logmans, H., Metselaar, R., \& Stacy, W. T. (1975). Optical measurement of magnetic anisotropy in thin garnet films. Applied Physics, 8(2), 143-150. https://doi.org/10.1007/BF00896031

DOI:

10.1007/BF00896031

Document status and date:

Published: 01/01/1975

\section{Document Version:}

Publisher's PDF, also known as Version of Record (includes final page, issue and volume numbers)

\section{Please check the document version of this publication:}

- A submitted manuscript is the version of the article upon submission and before peer-review. There can be important differences between the submitted version and the official published version of record. People interested in the research are advised to contact the author for the final version of the publication, or visit the $\mathrm{DOI}$ to the publisher's website.

- The final author version and the galley proof are versions of the publication after peer review.

- The final published version features the final layout of the paper including the volume, issue and page numbers.

Link to publication

\section{General rights}

Copyright and moral rights for the publications made accessible in the public portal are retained by the authors and/or other copyright owners and it is a condition of accessing publications that users recognise and abide by the legal requirements associated with these rights.

- Users may download and print one copy of any publication from the public portal for the purpose of private study or research.

- You may not further distribute the material or use it for any profit-making activity or commercial gain

- You may freely distribute the URL identifying the publication in the public portal.

If the publication is distributed under the terms of Article 25fa of the Dutch Copyright Act, indicated by the "Taverne" license above, please follow below link for the End User Agreement:

www.tue.nl/taverne

Take down policy

If you believe that this document breaches copyright please contact us at:

openaccess@tue.nl

providing details and we will investigate your claim. 
Appl. Phys. 8, 143-150(1975)

(C) by Springer-Verlag 1975

\title{
Optical Measurement of Magnetic Anisotropy in Thin Garnet Films
}

\author{
P. J. Rijnierse, H. Logmans, R. Metselaar, and W. T. Stacy \\ Philips Research Laboratories, Eindhoven, The Netherlands \\ Received 29 May 1975/Accepted 6 June 1975
}

\begin{abstract}
This paper describes an improved optical method for measuring locally the cubic and uniaxial magnetic anisotropy fields in thin garnet films. The derivative of the in-plane component of the magnetization is measured, using a double modulation technique which combines polarization modulation with field modulation. A simple graphical method is devised to calculate $H_{k}$ and $H_{u}$ from the extrema in this derivative curve. The results of measurements on magnetic garnet films obtained by different methods are compared. Local measurements of the anisotropy induced by substrate facet strain are described.
\end{abstract}

Index Headings: Magnetic anisotropy - Garnet films

Several methods have been proposed recently for measuring the magnetic anisotropy of thin garnet films. All of these measurements are based on either the direct observation of domain configurations $[1,2]$ or on a measurement of the Faraday rotation under certain external field conditions [3-7]. It is the latter method which we wish to discuss in this paper.

The methods proposed up to now have a number of drawbacks: poor signal-to-noise ratio, measurement of uniaxial and cubic anisotropy components together rather than separately, and a certain ambiguity in the deduction of anisotropy components from the measured curves.

In this paper we present a new version of this technique, designed to avoid these disadvantages. Essentially it employs modulation of both light polarization and external magnetic field, yielding a derivative signal of high signal-to-noise ratio. In order to obtain uniqueness of the resulting curve, the field component parallel to the sample plane is modulated. This also allows an independent measurement of the cubic anisotropy component. Detailed descriptions of the experimental technique and of the data analysis are given in Sections 1.1 and 1.2 , respectively.

In Section 2 the results obtained with this method are compared with ferrimagnetic resonance measurements and domain observations. Finally, in the last section, the capability of the method for measuring local changes in anisotropy is illustrated for the case of facet strain in the garnet substrate.

\section{Measuring Technique}

All existing methods have been worked out for the case when the sample plane is a (111) plane, and the anisotropy is a combination of a cubic component and an uniaxial contribution with the symmetry axis normal to the sample plane. We will also consider this particular case (which is that most frequently occurring in assessing bubble materials).

Krumme et al. $[3,4]$ measure the Faraday rotation $\theta_{F}$ of the sample by a standard polarization modulation technique as a function of external fields $H_{\perp}$ normal to and $H_{\|}$parallel to the sample plane ${ }^{1} . H_{\| /}$is applied along $[11 \overline{2}]$ or $[\overline{1} \overline{1} 2]$, so that the magnetization is always in the (110) plane. From $\theta_{F}$ they deduce plots of the angle $\vartheta$ between the magnetization and the sample normal as a function of $H_{\| /}$at fixed values of $H_{\perp}$. Their analysis, leading to values for the cubic and uniaxial

\footnotetext{
${ }^{1}$ Note that our indices $\perp$ and // agree with the convention of $[3,4]$, but differ from those of $[5-7]$. We feel that the chance of confusion is less when reference is made to the sample geometry.
} 
anisotropy fields, starts from the location of inflexion points in these plots. Therefore, it seems sensible to measure directly the derivative of such curves, where these points will show up as maxima or minima. This means that one tries to determine the "susceptibility" $\chi_{/ /}=d(M \cos \vartheta) / d H_{/ /}$, which is the derivative of the normal component of the magnetization direction.

In fact, the basis of the methods proposed by Josephs [5] and Shumate et al. $[6,7]$ is a susceptibility measurement, but since they modulate $H_{\perp}$, they measure $\chi_{\perp}$ rather than $\chi_{\| / /}$. Josephs [5] maintained a zero mean value of $H_{\perp}$ and measured only the tail of the susceptibility curve for high in-plane fields $H_{\| /}$. Shumate et al. $[6,7]$ employed a non-zero field $H_{\perp}$.

It is useful to go into the magneto-optical measurement in some detail in order to establish what is actually measured. Let the Faraday rotation of a specimen in a given magnetization state be $\theta_{F}$, and let polarizer and analyzer be at an angle $\alpha$ from the extinction position. Then the relative intensity of transmitted light is

$I^{*}=I / I_{0}=\sin ^{2}\left(\theta_{F}+\alpha\right)$.

Let a field component $H_{i}$ ( $i$ referring to either $\perp$ or $/$ components) be modulated at a frequency $\omega_{H}$ with (small) amplitude $\hat{H}$, then one has

$\theta_{F}=\theta_{F}(\bar{H})+\theta_{F}^{\prime}(\bar{H}) \hat{H} \sin \omega_{H} t+\ldots$

and to first order in $H$ one has

$I^{*}=\sin ^{2}\left(\theta_{F}+\alpha\right)+\sin \left[2\left(\theta_{F}+\alpha\right) \hat{H} \theta_{F}^{\prime}\right] \sin \omega_{r z} t+\ldots$

and at the frequency $\omega_{H}$ one detects a signal

$I_{H}^{*}=\sin \left[2\left(\theta_{F}+\alpha\right) \theta_{F}^{\prime} \hat{H}\right]$.

In practice, $\theta_{F} \ll 1$ and one can write

$I_{H}^{*}=\theta_{F}^{\prime} \hat{H}\left(\sin 2 \alpha+2 \theta_{F} \cos 2 \alpha\right)$.

This is the basis of the methods in [5-7]. The field $H_{\perp}$ is modulated and the signal $I_{H}^{*}$ is observed. From $(2 \mathrm{~b})$ it is clear that $I_{H}^{*}$ is only proportional to the desired quantity $\theta_{F}^{\prime}$ if $\alpha \gg \theta_{F}$. However, in that case there is a large dc component in the light intensity, as seen from (1), reducing the signal-to-noise ratio. If $\alpha$ is reduced to improve the signal-to-noise ratio, one measures a signal proportional to $\theta_{F} \cdot \theta_{F}^{\prime}$, in addition to that wanted. Thus, there is a conflict between the demands of low noise and disturbance-free signal, and one has to consider carefully what one is actually measuring.

To avoid this complication, we propose a double modulation method combining the polarization modulation of the standard technique employed in $[1,2]$ with the field modulation of $[3-5]$. Let the polarization angle $\alpha$ be modulated at frequency $\omega_{\alpha}$ and amplitude $\alpha, \alpha=\bar{\alpha}+\hat{\alpha} \sin \omega_{\alpha} t$. One can detect a signal $I_{\alpha}^{*}$ at frequency $\omega_{\alpha}$, which for small $\hat{\alpha}$ is equal to

$I_{\alpha}^{*}=\sin \left[2 \hat{\alpha}\left(\bar{\alpha}+\theta_{F}\right)\right]$.

If the field modulation frequency $\omega_{H} \ll \omega_{x}$, one can feed this signal to a second detector tuned to $\omega_{H}$ and detect a signal

$I_{\alpha H}^{*}=2 \hat{\alpha} \hat{H}\left(d \theta_{F} / d H\right) \cos 2\left(\theta_{F}+\bar{\alpha}\right)$.

Now $\theta_{F}$ is small and $\alpha$ can be chosen close to zero. Thus one has a signal $I_{\alpha H}^{*} \approx 2 \hat{\alpha} \hat{H}\left(d \theta_{F} / d H\right)$ of the desired form, while at the same time the dc intensity $I^{*} \approx \sin ^{2}\left(\alpha+\theta_{F}\right)$ is very small. In this way a low noise is combined with a pure derivative signal proportional to $d \theta_{F} / d H$. This derivative is proportional to the susceptibility we want to measure

$d \theta_{F} / d H_{i}=\theta_{F}(\vartheta=0) M d \cos \vartheta / d H_{i}=\theta_{F}(0) \chi_{i}$.

In such a double modulation scheme, it is of course important to make a proper choice of modulation frequencies and lock-in detector time constants. We have successfully employed two Brookdeal 401 A lockin detectors, with the polarization modulation at $1 \mathrm{kHz}$ and the corresponding time constant at $10 \mathrm{~ms}$, while for the field modulation we had $10 \mathrm{~Hz}$ and $1 \mathrm{~s}$, respectively. These are of course just typical values. The signal $I_{\alpha}$ is a measure of $\theta_{F}$ or $\cos \theta$, which can thus be monitored simultaneously.

Our proposed technique consists of measuring the susceptibility $\chi_{/ /}$as a function of $H_{/ /}$for positive and negative values of $H_{/ /}$oriented along a [112] direction. The sample is oriented using a Laue transmission diffraction pattern, which is displayed on a TV monitor for ease of operation. The perpendicular field $H_{\perp}$ is kept at a value high enough to avoid both domain formation and instabilities [3] in the uniform magnetization configuration. The susceptibility plot shows extrema for both signs of $H_{/ /}$. The asymmetry in the peaks is a measure of the ratio of cubic to uniaxial anisotropy, and combined with their location yields values for these anisotropy fields. In addition, by scanning $\chi_{/ /}$as a function of $H_{\perp}$ for values of $H_{/ /}$larger than the anisotropy fields, an independent check on the cubic anisotropy is possible. The analysis of the measurements, which can be done either graphically or by a numerical fit, is discussed in the next section.

One important advantage of this technique is that the anisotropy fields can be determined uniquely from the positions of the susceptibility peaks alone, leaving agreement between theoretical and experimental curve 
shapes as a further check on the correctness of the analysis. In the method of Shumate et al. [6,7], these positions are not unique: different combinations of anisotropy fields can give rise to the same peak positions. It then becomes necessary to use curve shape as well to avoid erroneous conclusions. In their technique, moreover, it is necessary to rotate the sample, in order to measure with $H_{/ /}$along various crystal directions in the plane. This makes it impossible to carry out local measurements, unless the spot investigated is exactly on the axis of rotation. As in the method of Krumme et al. [3, 4], after initial orientation we do not need any further sample rotation; thus local measurements using a narrow light beam are feasible. An example of such a measurement is discussed in Section 3.

\section{Theory and Analysis of Measurements}

In this section we derive a description of the shape of susceptibility vs in-plane field curves in terms of external and anisotropy fields. From this we construct a simple graphical method for the analysis of experimental results; alternatively one can employ a numerical fit to the observed peak positions.

In this paper, all energies will be divided by the saturation magnetization $M$, and the discussion is essentially in terms of anisotropy fields. The sample is oriented with [111] as the sample normal, $H_{\| /}$being applied in the (110) plane and counted positive when

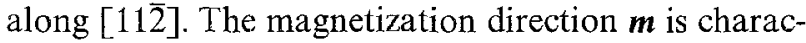
terized by a polar angle $\vartheta$ measured from [111] and an azimuthal angle $\varphi$ measured from [112̄] (Fig. 1).

With this sample orientation, any strain- or growthinduced anisotropy is expected on symmetry grounds to have uniaxial symmetry. Anisotropy fields are defined as follows: an "effective uniaxial" field $H_{e}=$ $2 K_{u} / M-4 \pi M$ is built up from uniaxial anisotropy $\left(K_{u}\right)$ and demagnetization energy $\left(2 \pi M^{2}\right)$ contributions, and the cubic field is $H_{k}=2 K_{1} / M$. In terms of these fields, the reduced total energy density for uniform magnetization along $(\vartheta, \varphi)$ can be written in the form

$$
\begin{aligned}
E= & -H_{\perp} \cos \vartheta-H_{/ /} \sin \vartheta+E^{a}(\vartheta, \varphi), \\
E^{a}= & \frac{1}{2} H_{e} \sin ^{2} \vartheta+\frac{1}{24} H_{k} \\
& \cdot\left(-8 \sin ^{2} \vartheta+7 \sin ^{4} \vartheta+4 \sqrt{2} \sin ^{3} \vartheta \cos \vartheta \cos 3 \varphi\right) .
\end{aligned}
$$

Here we have introduced $E^{a}$, the reduced anisotropy energy density; for other sample geometries, $E^{a}$ will change, but the formulation in terms of $E^{a}$ remains similar.

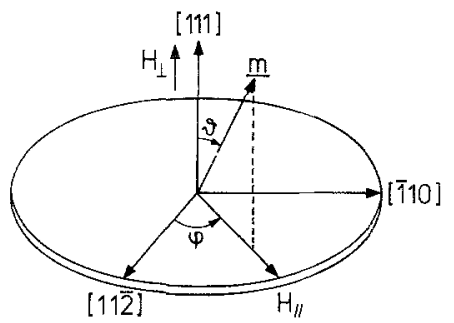

Fig. 1. Sample geometry

In order to find the equilibrium magnetization direction, we require the derivative of $E$ to be zero along two mutually orthogonal directions. We define these directions as follows: if $\boldsymbol{m}$ is a unit vector along the magnetization direction $(\vartheta, \varphi)$, then $\boldsymbol{g}$ is a unit vector perpendicular to $m$ in the sample plane along $(\pi / 2, \varphi-\pi / 2)$. The third orthogonal direction is along $\boldsymbol{h}=\boldsymbol{m} \times \boldsymbol{g}$. Small deviations can be written as

$\boldsymbol{m}+d \boldsymbol{m}=\boldsymbol{m} \sqrt{1-\delta^{2}-\varepsilon^{2}}+\delta \boldsymbol{g}+\varepsilon \boldsymbol{h}$,

and the required derivatives are those with respect to $\delta$ and $\varepsilon$. It can be seen that varying $\varepsilon$ alone means a change in $\vartheta$, while variation of $\delta$ refers to a motion of $\boldsymbol{m}$ perpendicular to the (110) plane. Some algebra shows that derivatives with respect to $\delta$ and $\varepsilon$ can be expressed in those with respect to $\delta$ and $\varphi$

$$
E_{\delta}=-E_{\varphi} / \sin \vartheta, \quad E_{\varepsilon}=E_{\vartheta} ;
$$

$E_{\delta \delta}=E_{\varphi \varphi} / \sin ^{2} \vartheta+E_{\vartheta} \operatorname{cotg} \vartheta, \quad E_{\varepsilon \varepsilon}=E_{\vartheta \vartheta}$,

$E_{\delta \varepsilon}=-E_{\vartheta \varphi} / \sin \vartheta+E_{\varphi} \cos \vartheta / \sin ^{2} \vartheta$.

In equilibrium we require $E_{\varepsilon}=E_{\delta}=0$ with the stability conditions $E_{\varepsilon \varepsilon}>0, E_{\delta \dot{\delta}} E_{\varepsilon \varepsilon}-E_{\delta \varepsilon}^{2}>0$. For magnetization directions in the (110) plane, where $\varphi=0$, one has always $E_{\delta} \equiv 0, E_{\delta \varepsilon} \equiv 0$ for any $\vartheta$. Thus, with $H_{/ /}$in the (110) plane, $\boldsymbol{m}$ lies in that plane too, provided of course the stability criteria are met. Thus equilibrium directions are determined by

$0=E_{\varepsilon}=H_{\perp} \sin \vartheta-H_{/ /} \cos \vartheta+E_{\varepsilon}^{a}$,

provided

$0<E_{\delta \delta}=H_{\perp} \cos \vartheta+H_{/ /} \sin \vartheta+E_{\delta \delta}^{a}$,

$0<E_{\varepsilon \varepsilon}=H_{\perp} \cos \vartheta+H_{/ j} \sin \vartheta+E_{\varepsilon \varepsilon}^{a}$.

Here the first of the conditions (8b) refers to stability against small deviations out of the (110) plane, while the second refers to a similar stability within that plane. 
The derivatives of $E^{a}$ in (8) can be written explicitly for our geometry, with $\varphi=0$, as

$$
\begin{aligned}
E_{\varepsilon}^{a}= & H_{e} \sin \vartheta \cos \vartheta+\frac{1}{6} H_{k} \\
& \cdot\left[\sin \vartheta \cos \vartheta\left(-4+7 \sin ^{2} \vartheta\right)+\sqrt{2} \sin ^{2} \vartheta\left(3-4 \sin ^{2} \vartheta\right)\right], \\
E_{\partial \delta}^{a}= & H_{e} \cos ^{2} \vartheta+\frac{1}{6} H_{k}\left[-4+11 \sin ^{2} \vartheta\right. \\
& \left.-7 \sin ^{4} \vartheta+\sqrt{2} \sin \vartheta \cos \vartheta\left(-6-4 \sin ^{2} \vartheta\right)\right], \\
E_{\varepsilon \varepsilon}^{a}= & H_{e}\left(1-2 \sin ^{2} \vartheta\right)+\frac{1}{6} H_{k}\left[-4+29 \sin ^{2} \vartheta\right. \\
& \left.-28 \sin ^{4} \vartheta+\sqrt{2} \sin \vartheta \cos \vartheta\left(6-16 \sin ^{2} \vartheta\right)\right] .
\end{aligned}
$$

The equilibrium condition (8a) gives the functional dependence of $\vartheta$ on $H_{\perp}$ and $H_{/ /}$, though this is not explicitly expressible unless $H_{\perp}=0, H_{k}=0$. The susceptibilities $\chi_{\perp}$ and $\chi_{/ /}$are found by differentiating (8a) with respect to $H_{\perp}$ or $H_{/ /}$, respectively. Thus one finds $\chi_{i}=\sin \vartheta\left(\partial E_{\varepsilon} / \partial H_{i}\right) /\left(\partial E_{\varepsilon} / \partial \vartheta\right)$.

Since $\partial E / \partial \vartheta=E_{\varepsilon \varepsilon}$ one obtains from (8a)

$\chi_{/ /}=-\sin \vartheta \cos \vartheta / E_{\varepsilon \varepsilon}(\vartheta), \quad \chi_{\perp}=\sin ^{2} \vartheta / E_{\varepsilon \varepsilon}(\vartheta)$,

where of course $\vartheta$ is a function of $H_{\perp}$ and $H_{/ /}$through (8a). In previous methods proposed $3,4,5,6,7$ scans are made of $\chi_{\perp}$ or $\chi_{/ /}$as a function of $H_{/ /}$for fixed $H_{\perp}$. Peaks in plots of $\chi_{\perp}$ or $\chi_{/ /}$are found by differentiation of (10). Explicitly, the problem reduces to finding an angle $\hat{\vartheta}$ satisfying

$\left[E_{\vartheta 99}^{a} \cos ^{2} \vartheta+E_{\vartheta}^{a} D_{i}\right] \sin \hat{\vartheta}+\left[E_{\vartheta \vartheta}^{a} \cos \hat{\vartheta}+H_{\perp}\right]$

$\left(D_{i}-\cos ^{2} \hat{\vartheta}\right)=0$,

where $D_{\perp}=2 \sin ^{2} \hat{\vartheta}-1$ for peaks in $\chi_{\perp}, D_{\| /}=2 \sin ^{2} \hat{\vartheta}$ for peaks in $\chi_{/ /}$. Subsequently, the corresponding peak positions $H_{/ /}$are found by substituting this value for $\hat{\vartheta}$ into (8a).

Suitable experimental restrictions on $H_{\perp}$ are such that the stability conditions (8b) are never violated during an entire scan of $H_{/ /}$from $-\infty$ to $+\infty$. By eliminating $H_{\| /}$from (8b) by means of (8a), one can determine a minimum value $H_{m}$ for $H_{\perp}$ for any combination of $H_{e}$ and $H_{k}$. Provided $H_{\perp}$ is chosen larger than $H_{m}$, instabilities will not occur. Of course, this reasoning presupposes a uniform magnetization: conditions to prevent domain formation will have to be met separately. In Fig. 2 we have plotted values for $H_{m}$ for all possible combinations of $H_{e}$ and $H_{k}$. Drawn lines refer to values of $H_{m} /\left|H_{k}\right|$ while dashed lines refer to values of $H_{m} /\left|H_{e}\right|$. In the upper half of the figure values of $H_{m}$ are given for $H_{e}>0$ as a function of $H_{k} / H_{e}$. For negative $H_{e}$, meaning that $K_{u}<0$ or that $4 \pi M$ is larger than $2 K_{u} / M$, we have plotted values in the lower half

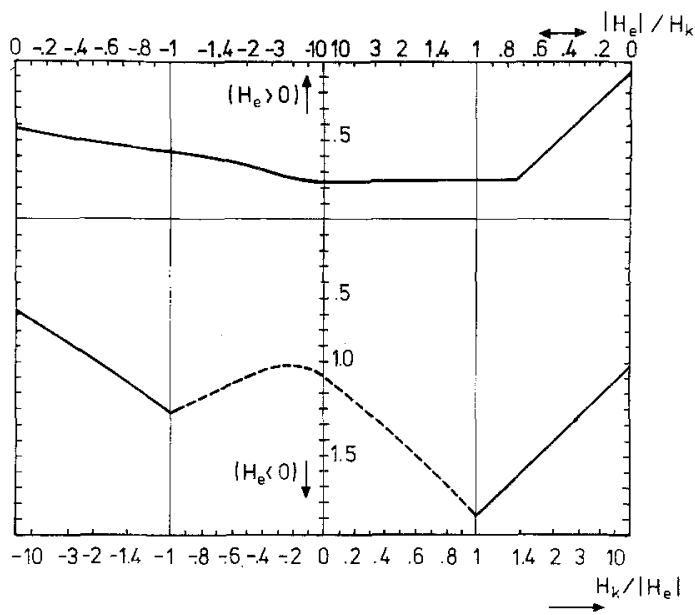

Fig. 2. Minimum values $H_{m}$ of the perpendicular field necessary to obtain stable solutions for all possible combinations of $H_{e}$ and $H_{k}$. (Drawn lines: $H_{m} /\left|H_{k}\right|$, dashed lines: $H_{m} /\left|H_{\mathrm{e}}\right|$.) In the upper half $H_{m}$ values are given for $H_{e}>0$, in the lower half for $H_{e}<0$. Note: horizontal scale is linear in $H_{k} /\left|H_{e}\right|$ for $\left|H_{k} / H_{e}\right|<1$, and inversely linear otherwise

of Fig. 2 as a function of $H_{k} /\left|H_{e}\right|$. Thus the right-hand side of Fig. 2 refers to positive, the left-hand side to negative $H_{k}$. Once an initial guess as to the values of $H_{e}$ and $H_{k}$ has been made, Fig. 2 can be used to find experimental conditions where no instabilities will occur [8].

To make such a preliminary guess of $H_{k}$, we propose a simple procedure for finding $H_{k}$, independent of the value of $H_{e}$. From (10) it is apparent that $\chi_{/ /}$will change $\operatorname{sign}$ when either $\sin \vartheta$ or $\cos \vartheta$ changes sign. An obvious sign change occurs when $H_{/ /}=0$. However, it is possible to have $\cos \vartheta=0, \vartheta= \pm \pi / 2$ provided

$H_{\perp} \sin \vartheta=-E_{\varepsilon}^{\alpha}(\cos \vartheta=0)=-\frac{\sqrt{2}}{6} H_{k}$.

Stability is assured if $H_{/ /} \sin \vartheta>H_{e}+\frac{1}{2} H_{k}$. Thus, provided $H_{/ /}$is large enough to maintain stability, one will find a zero crossing in $\chi_{/ /}$when $H_{\perp}$ is varied while $H_{/ /}$is kept fixed. The value of $H$ for which $\chi_{/ /}=0$ is a measure of $H_{k}$, independent of the values of $H_{/ /}$employed and independent of $H_{e}$. Incidentally, such a simple check is not possible when measuring $\chi_{\perp}$, since that shows at best a shallow extremum. This check is especially valuable for small values of the ratio $H_{k} / H_{e}$, since it then is difficult to measure $H_{k}$ accurately from the asymmetry in the susceptibility plot alone.

Another simple check consists in observing the slope of the $\chi_{1 /}$ vs $H_{1 /}$ plots at zero field. Here one should have

$d \chi_{/ /} / d H_{/ /}=\left[H_{\perp}+H_{e}-\frac{2}{3} H_{k}\right]^{-2}, \quad H_{/ /}=0$, 


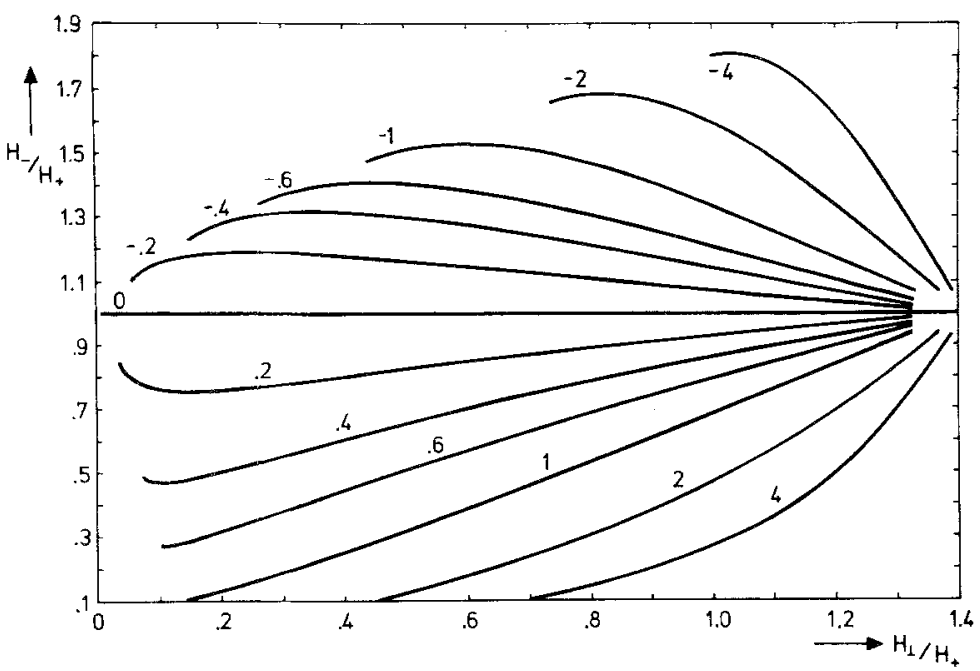

Fig. 3a. $H_{-} / H_{+}$as a function of $H_{\perp} / H_{+}$for $H_{e}>0$. (Parameter: $\left.H_{k} / H_{e}\right)$

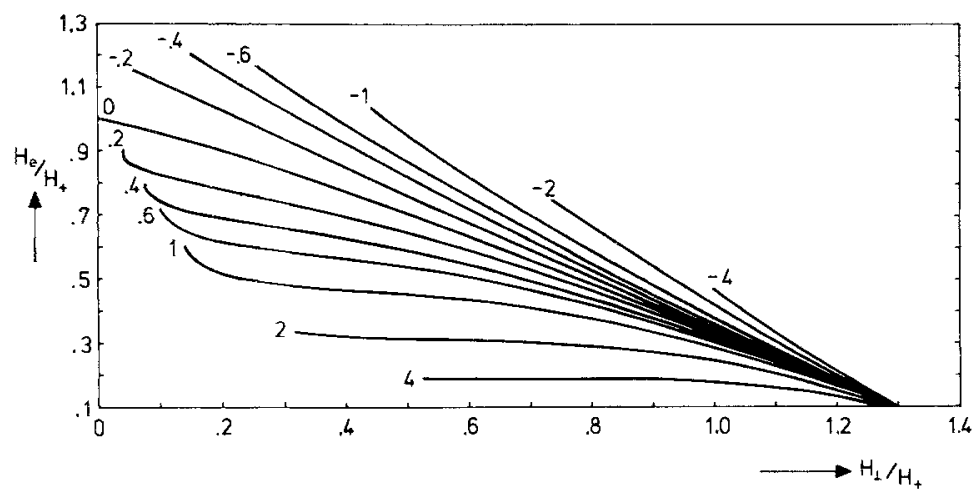

Fig. 3b. $H_{e} / H_{+}$as a function of $H_{\perp} / H_{+}$for $H_{e}>0$. (Parameter: $H_{k} / H_{e}$ )

affording a further test of the consistency of the results obtained.

The main analysis, however, will have to come from an analysis of the measured positions $H_{+}$(for positive $H_{/ /}$) and $H_{-}$(for negative $H_{l}$ ) of the peaks in the susceptibility vs in-plane field plots. Given $H_{\perp}$ and the corresponding fields $H_{+}$and $H_{-}$one can make a numerical fit to yield values of $H_{e}$ and $H_{k}$ which lead to these peak positions. It is then convenient, as a further consistency check, to plot the curves of $\chi_{/ 1}$ vs $H_{\| /}$ predicted from these calculated values, since the complete curve shapes should agree.

For a quicker analysis, which can if necessary always be complemented by numerical methods, a simple graphical method has been devised (Fig. 3). This is a natural extension of the use of (Ref. [6], Fig. 2). It rests on the observation that all equations employed are linear in the fields so that universal curves can be con- structed by plotting ratios of fields rather than the fields themselves. Experiments yield fields $H_{\perp}, H_{+}$, and $H_{-}$; in the analysis given by Shumate et al. [6] cubic anisotropy is neglected and they plot $H_{+} / H_{e}$ as a function of $H_{\perp} / H_{+}$(in our notation). Similar plots for nonzero cubic anisotropy give a collection of curves for different values of the ratio $H_{k} / H_{e}$. Note that we have plotted $H_{e} / H_{+}$in contrast to the convention employed in [6]. The ratio $H_{k} / H_{e}$ can be determined from a plot of $H_{-} / H_{+}$as a function of $H_{1} / H_{+}$, again giving a set of curves with $H_{k} / H_{e}$ as a parameter. In Figs. $3 \mathrm{a}$ and $3 \mathrm{~b}$ we show such a combination of curves, drawn under the assumption that $H_{e}>0 ;$ Figs. $3 \mathrm{c}$ and $3 \mathrm{~d}$ give similar curves for $H_{e}<0$. We have used $H_{+} / H_{\perp}$ as the abscissa in Figs. 3c, d, since in the latter case $H_{\perp} / H_{+}$is always larger than $\sqrt{2}$.

Figure 3 reveals two advantages of this method: (i) except for values of $H_{\perp}$ immediately above the minimum 


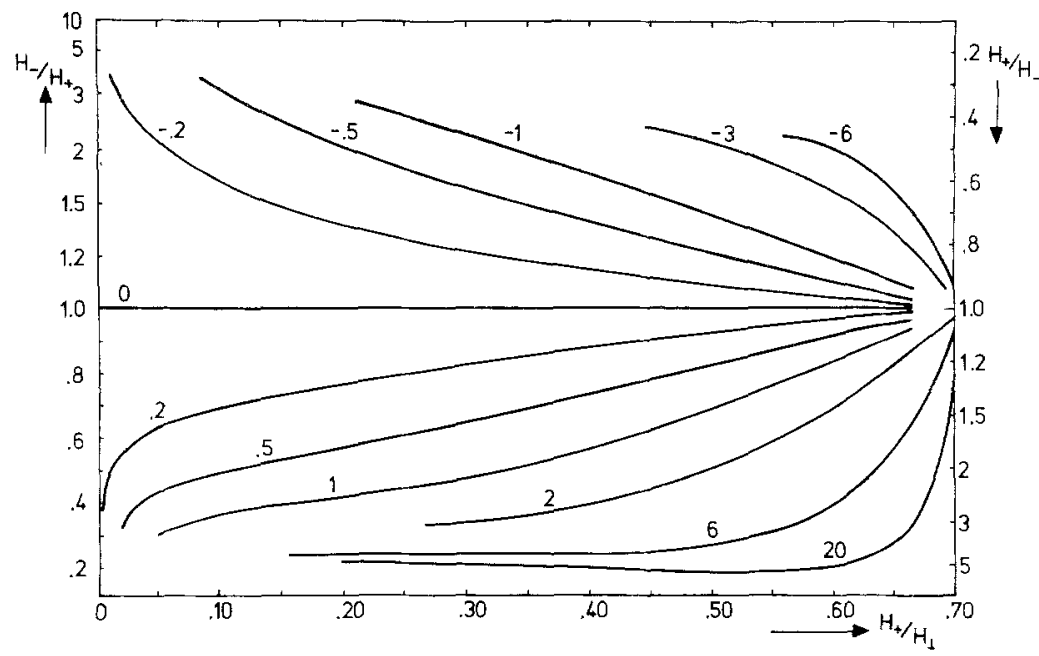

Fig. 3c. $H_{-} / H_{+}$as a function of $H_{+} / H_{\perp}$ for $H_{e}<0$. (Parameter: $\left.H_{k} /\left|H_{e}\right|\right)$

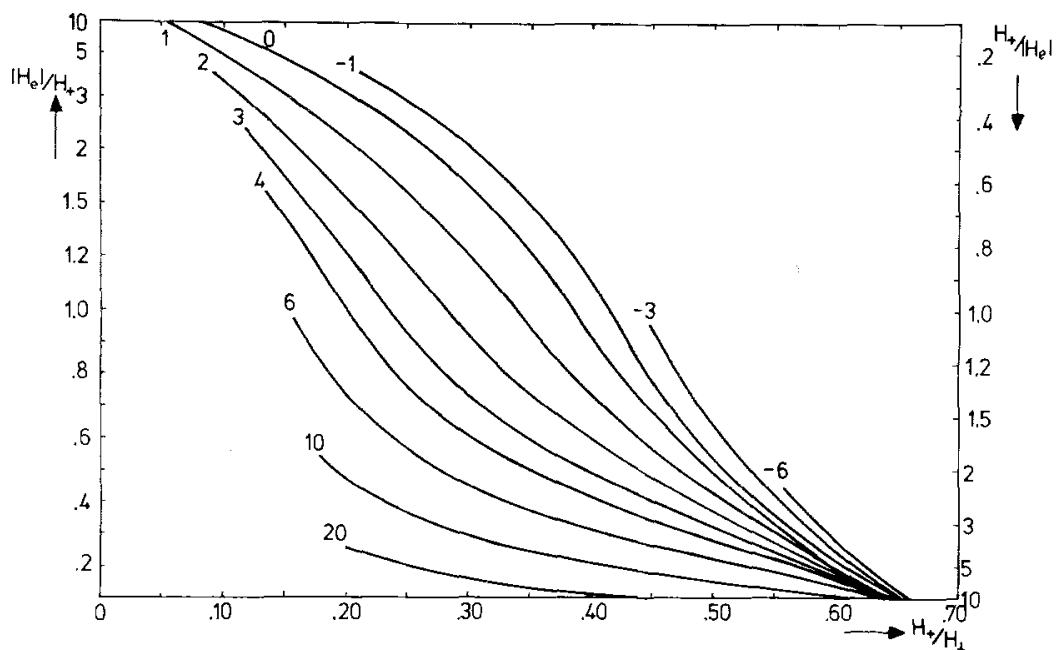

Fig. 3d. $\left|H_{e}\right| / H_{+}$as a function of $H_{+} / H_{\perp}$ for $H_{e}<0$. (Parameter: $H_{k} /\left|H_{e}\right|$ ) Note: vertical scales in Figs. 3c/d are linear in the lower half, inversely linear in the upper half of the graphs

value $H_{m}$, the curves do not cross, and a unique determination may be made from the positions $H_{+}$and $H_{-}$alone; (ii) the regions for $H_{e}$ positive and negative are completely separated except for the common point $H_{\perp} / H_{+}=\sqrt{2}, H_{-} / H_{+}=1$, which is reached only for $H_{\perp} \rightarrow \infty$; thus from the observed value of $H_{\perp} / H_{+}$the sign of $H_{e}$ may immediately be ascertained. (Similar plots for $\chi_{\perp}$ peaks show that these two advantages are lost with perpendicular field modulation.)

Thus our proposed measuring cycle contains the following steps after sample orientation and selection of the area to be viewed (it is assumed that $H_{k}<0$ ):

(a) for a value of $H_{\perp}$ large enough to collapse all domains when $H_{/ /}=0$, we scan $\chi_{/ /}$as a function of
$H_{1 /}>0$; the peak position gives a rough indication of the magnitude of $H_{e}+\frac{1}{2} H_{k}$;

(b) for a value of $H_{1 /}<0$, in absolute value larger than that found under (a), we scan $\chi_{/ /}$as a function of $H_{\perp}$; the zero crossing gives $H_{k}$ according to (11);

(c) a "safe" value of $H_{\perp}>H_{m}$ is determined from Fig. 2 and the preliminary values of $H_{k}$ and $H_{e}$ found above; for this value a plot of $\chi_{/ /}$is made with $H_{/ /}$varying from large negative to large positive fields; from the peak positions $H_{+}$and $H_{-}$we determine $H_{e}$ and $H_{k}$ either graphically or numerically;

(d) the results of (b) and (c) are compared for consistency; "theoretical" susceptibility plots are compared with the experimental ones both regarding the 
general shape and with respect to the slope at the origin.

As mentioned in Section 1.1, we have only discussed the case of a (111) sample plane. Of course, the method is also applicable to cases with a different sample orientation. For example, for a (110) sample plane, stable solutions can be found with $H_{/ /}$along a [00̄] direction or along a [ [110] direction, while for a (001) sample plane $H_{/ /}$can be suitably chosen along a [100] direction. Due to the higher symmetry in these three cases, $H_{+}=H_{-}$and as a consequence an independent measurement of $H_{k}$ is necessary to determine $H_{e}$ from the extremum in $\chi_{/}$.

\section{Comparison with Domain Observations and FMR Measurements}

The optical method described above has been employed to determine the anisotropy parameters of a number of samples with different compositions. In Table 1 examples are shown of four garnet films with ratios of $K_{1} / K_{u}$ varying between about 0.1 and 1 . The films used for these measurements were grown by liquid phase epitaxy on (111) oriented gadolinium gallium garnet substrates. The approximate compositions are given in Table 1 . The $K_{u}$ and $K_{1}$ (fit) data were obtained from a fit of the experimentally determined values of $H_{+}$and $H_{-}$, using the method described in Section 1. The $K_{1}$ (direct) values given in the table were calculated from the zero crossing of $\chi_{/ /}$ as a function of $H_{\perp}$ at a fixed value of $H_{\|}$[cf. (11)]. For comparison we also show the anisotropy values obtained from FMR measurements at $10 \mathrm{GHz}$ and from an observation of the in-plane field $H_{\| /}^{*}$ at which the domain contrast disappears [1]. The anisotropy field $H_{k}$ used to calculate the $K_{u}$ (domain) values given in Table 1 was obtained from $H_{/ /}^{*}$ by applying the correction described by Druyvesteyn et al. [9]. Measurements of the saturation magnetization were performed with a vibrating sample magnetometer.

From Table 1 we conclude the following:

1) There is good agreement between the $K_{1}$ values obtained directly from the zero crossing of $\chi_{/ /}$versus $H_{\perp}$ and the values from the curve fitting of $\chi_{/ /}$versus $H_{/ /}$.

2) There is also good agreement between the results obtained by the optical method and by FMR. The uncertainty in $K_{1}$ is, of course, largest for small $K_{1} / K_{u}$ ratios in both methods.

3) The $K_{u}$ values calculated from the domain observation method are in good agreement with those found with the other two techniques. Since these $K_{u}$ values are not corrected for the $K_{1}$ contribution they contain, such agreement is expected only for small $K_{1} / K_{u}$ (as in the case of specimen 3). For specimen 2, however, where $K_{1} / K_{u}=1$, this agreement seems purely accidental.

\section{Local Measurement of the Anisotropy - The Effect of Substrate Facet Strain}

Facet strain is a common garnet substrate defect which is associated with the formation of facets on the solidliquid growth interface. When a garnet slice containing a facet region is used as a substrate for a magnetic

Table 1. Comparison of uniaxial anisotropy constant $K_{u}$ and cubic anisotropy constant $K_{1}$ obtained by different experimental methods

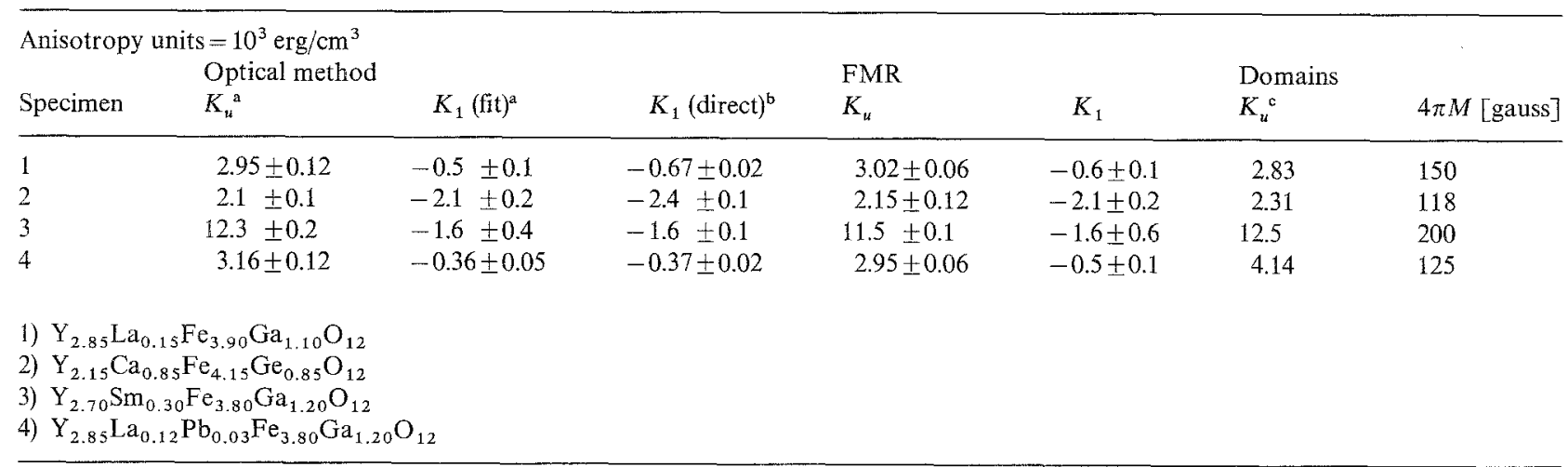

${ }^{a}$ Calculated from the position of the extrema in the $\chi_{/ /}$vs $H_{/ /}$curve.

${ }^{\mathrm{b}}$ Calculated independently from the zero crossing of the $\chi_{/ /}$vs $H_{\perp}$ curve.

${ }^{c}$ Calculated from the field $H_{/ /}^{*}$ at which the domain contrast vanished. 


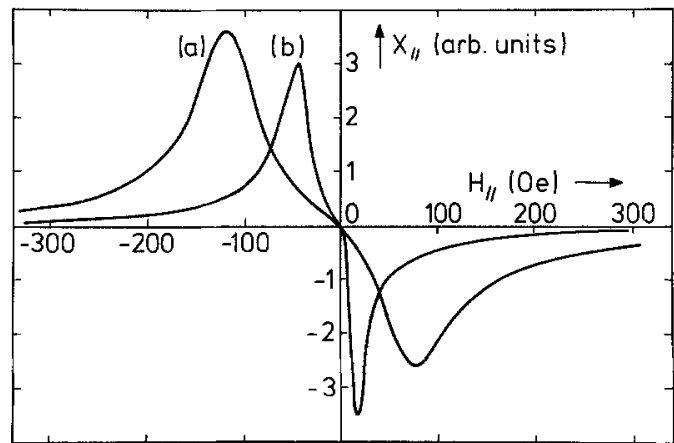

Fig. 4. Measured curve of $\chi_{/ 1}$ vs. $H_{1 /}$ for a garnet film $(4 \pi M=16$ gauss) grown on a $\mathrm{Gd}_{3} \mathrm{Ga}_{5} \mathrm{O}_{12}$ substrate containing facet regions. Curve (a): local measurement within a facet region. Curve (b): local measurement outside a facet region

layer, the associated strain is replicated by the layer $[10,11]$ and has been found to cause a local change in the magnetic anisotropy $[4,11,12]$. A direct measurement of this anisotropy change is illustrated in Fig. 4 for a facet region approximately $1 \mathrm{~mm}$ in diameter. The layer composition chosen for this measurement was $\mathrm{Y}_{2.85} \mathrm{La}_{0.15} \mathrm{Fe}_{3.75} \mathrm{Ga}_{1.25} \mathrm{O}_{12}$. For this composition both the $4 \pi M$ and $K_{u}$ are small and thus the effect of facet strain is relatively large compared to that of layers with larger anisotropies. This is clearly evident in the plot of $\chi_{/ /}$versus $H_{/ /}$in Fig. 4. Here it can be seen that the susceptibility peaks were shifted to larger $H_{/ /}$ values as the focussed light beam was moved from the unstrained region to the facet strain area of the magnetic layer. The measured values of $K_{u}$ and $K_{1}$ were -26 and $-35 \mathrm{erg} / \mathrm{cm}^{3}$ outside the facet region and +33 and $-31 \mathrm{erg} / \mathrm{cm}^{3}$ inside the facet region. The measured $4 \pi M$ was 16 gauss.

The facet strain replicated by the layer was measured with a double crystal diffractometer and found to be $1.3 \times 10^{-4}$. From this and the above values for $K_{u}$, one can estimate the magnetoelastic coupling constant associated with the change in anisotropy. For a (111) layer with a biaxial planar strain $\varepsilon$ the induced anisotropy $\Delta K_{u}$ is

$\Delta K_{\mathrm{u}}=\frac{3}{2} C \lambda_{111}$

where $C=C_{11}+C_{12}+2 C_{44}$ is the appropriate elastic constant and $\lambda_{111}$ the magnetostriction constant. From the above values for $\Delta K_{u}$ and $\varepsilon$ we arrive at $C \lambda_{111}=$ $3.0 \times 10^{5} \mathrm{erg} / \mathrm{cm}^{3}$ for this particular composition. This is in reasonable agreement with values estimated from published data on the elastic [13] and magnetostriction [14] constants of the Ga substituted iron garnets.

\section{Conclusion}

Using a combined polarization modulation and field modulation technique a derivative signal of the Faraday rotation is obtained with a high signal-tonoise ratio. This derivative, which is proportional to $\chi_{/}$, is measured as a function of $H_{/ /}$for negative and positive values of $H_{/ /}$along a [11 $\left.\overline{2}\right]$ direction. Using the position of the extrema of this curve as input data one can calculate the uniaxial and cubic anisotropy fields with the aid of either a graphical method or a numerical fit. From the zero crossing of $\chi_{/ /}$as a function of $H_{\perp}$ at a fixed value of $H_{/ /}$one can obtain an independent measurement of the cubic anisotropy constant. The method is applicable both for positive and negative values of the anisotropy constants. The data obtained are in good agreement with FMR data.

Since the sample is fixed in space, local anisotropy measurements can be carried out by using a focussed light beam. To illustrate such a measurement the change in layer anisotropy due to facet strain in the substrate has been measured and the magnetoelastic coupling constant estimated.

Acknowledgements. We wish to thank B. Hoekstra for performing the FMR measurements, J. Haisma for the domain observation measurements and W. Tolksdorf(Philips Forschungslaboratorium Hamburg) and J.M.Robertson for providing the garnet films.

\section{References}

1. A.J.Kurtzig, F.B.Hagedorn: IEEE Trans. Mag. MAG-7, 473 (1971)

2. A.Hubert, A.P.Malozemoff, I.C. De Luca: J. Appl. Phys. 45, 3562 (1974)

3. J. P. Krumme, P.Hansen, J.Haberkamp: Phys. Stat. Sol. (a) 12 , $483(1972)$

4. P. Hansen, J.P. Krumme: J. Appl. Phys. 44, 2847 (1973)

5. R.M. Josephs: A.I.P. Conf. Proc. 10, 286 (1973)

6. P.W.Shumate Jr., D.H.Smith, F.B.Hagedorn: J. Appl. Phys. 44, 449 (1973)

7. P.W.Shumate Jr.: J. Appl. Phys. 44, 3323 (1973)

8. In the derivation of the data for the (theoretical) plot in [Ref. 7, Fig. 6], apparently the stability criteria (8b) were not imposed. For higher values of $\left|K_{1}\right|$ and $\varphi=60^{\circ}$ in the notation of [7], these conditions are not satisfied, and as a consequence a singularity rather than an extremum in the susceptibility plot should be found, and part of Fig. 6, curve a) should not exist as a stable solution

9. W.F.Druyvesteyn, J.W. Dorleijn, P.J.Rijnierse: J. Appl. Phys. 44, 2397 (1973)

10. W.T.Stacy, U.Enz: IEEE Trans. Mag. MAG-8, 268 (1972)

11. H.L.Glass, P.J.Besser, T.N.Hamilton, R.L.Stermer: Mater. Res. Bull. 8, 309 (1973)

12. G.P.Gill, R.J.Fairholme: J. Mat. Sci. 8, 1115 (1973)

13. S. Haussühl, D. Mateika: Z. Naturforsch. 27a, 1522 (1972)

14. P. Hansen: J. Appl. Phys. 45, 3638 (1974) 